\title{
Application of USP Apparatus 7 to In Vitro Drug Release in Scopolamine Transdermal Systems
}

Marilyn X. Zhou' ${ }^{1}$, Duane Shoudt,

e-mail:marilyn.zhou@avevadds.com Genaro Calderon, and Min Feng

Analytical Research and Development, Aveva Drug Delivery

Systems, Inc., 3250 Commerce Parkway, Miramar, FL

33025 USA

\section{Abstract}

To monitor in vitro drug release in scopolamine transdermal systems, the USP Apparatus 7 was selected. However, there are very few manufacturers that make USP Apparatus 7. In this article, we studied the in vitro scopolamine drug release test using a newly designed USP Apparatus 7 from Logan Instruments, Model RRT7 , and compared it with a USP Apparatus 7 system made by Varian. The results demonstrate that the drug release profiles obtained using the two USP Apparatus 7 systems are equivalent. The drug release profiles obtained using the two 12-sample carousels of the Logan System are also equivalent. Repeatability was measured on the Logan instrument and found acceptable.

\section{Introduction}

F

or a transdermal system, the drug release test is

important for monitoring batch-to-batch reproducibility and manufacturing deviations for quality control (QC) purposes. However, the approach and purpose of performing the drug release test may not necessarily be the same between Research and Development and QC (1), particularly for a transdermal system. To obtain a reliable prediction of bioavailability for a new drug application or bioequivalence for an abbreviated new drug application, the Franz diffusion cell with human cadaver skin is often used, which is desirable for the prediction of in vivo performance of the drug product. For QC purposes and a more economical

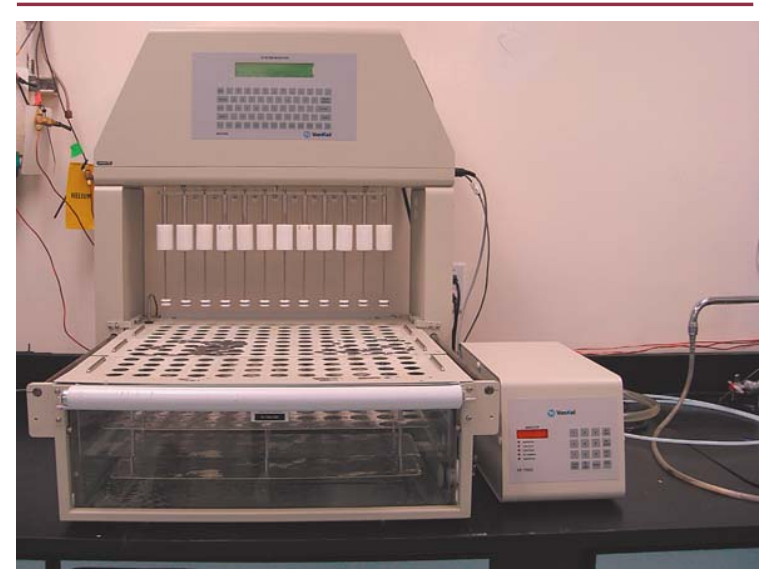

Figure 1. USP Apparatus 7 and the temperature controller (VK 750D Heater/Circulator) made by VanKel/Varian.

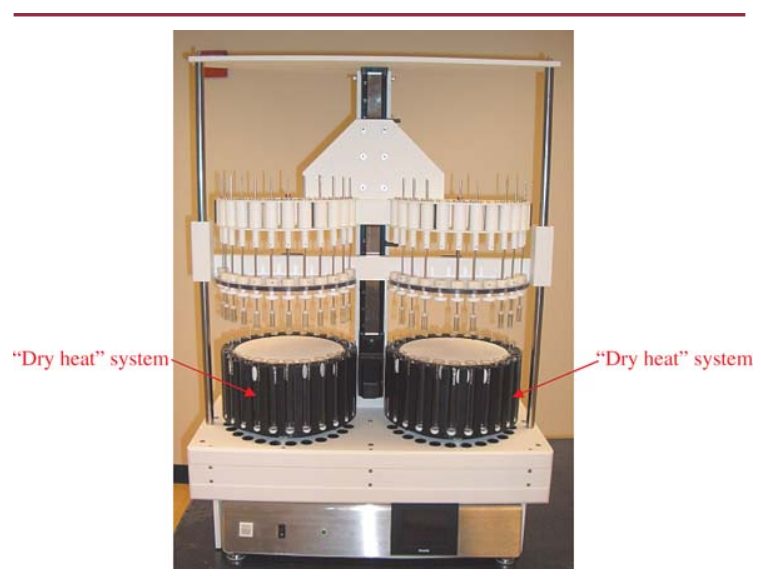

Figure 2. USP Apparatus 7 and the temperature controller made by Logan Instruments.

approach, a USP Apparatus 5 (Paddle over Disc), 6 (Cylinder), or 7 (Reciprocating Holder) can be used for the determination of drug release rate of transdermal systems.

The scopolamine transdermal system is a circular, flat patch designed for continuous release of scopolamine following application to an area of intact skin on the head, behind the ear. The patch is used for prevention of nausea and vomiting associated with motion sickness and recovery from anesthesia and surgery (2). To test the in vitro drug release in scopolamine transdermal systems, USP Apparatus 7 was used. The design of USP Apparatus 7 is described in the current United States Pharmacopeia (3). The assembly consists of a set of volumetrically calibrated or tared solution containers made of glass or other suitable inert material, a motor and drive assembly to reciprocate the system vertically and to 
Table 1. Dipping Program and Sampling Times.

\begin{tabular}{|l|c|c|}
\hline \multicolumn{2}{|c|}{ Description of Parameter } & Range or Value \\
\hline \multirow{5}{*}{ Dipping Time: } & Row 1 & 1 hour \\
\cline { 2 - 3 } & Row 2 & 1 hour \\
\cline { 2 - 3 } & Row 3 & 2 hours \\
\cline { 2 - 3 } & Row 4 & 2 hours \\
\cline { 2 - 3 } & Row 5 & 18 hours \\
\cline { 2 - 3 } & Row 6 & 24 hours \\
\cline { 2 - 3 } & Row 7 & 24 hours \\
\hline Sampling Times & & $1,2,4,6,24,48$, and 72 hours \\
\hline
\end{tabular}

index the system horizontally to a different row of vessels automatically, if desired, and a set of suitable sample holders. The solution containers are partially immersed in a suitable water bath of any convenient size that permits maintaining the temperature.

The sources of USP Apparatus 7 are very limited. One of the manufacturers is VanKel/Varian. Its design is the same as that described in the USP, shown in Figure 1. There are 12 sample tubes that automatically traverse up to 12 rows. Temperature is controlled using the VK 750D Heater/Circulator within a water bath (4). Its physical dimension is about 34 " $\times 28$ " $\times 32$ " (width $\times$ depth $\times$ height) plus a sink for water circulation. A plastic sheet that loosely covers the test tubes is used to minimize evaporation. Recently, Logan Instruments Corp. introduced a novel design of USP Apparatus 7, Model RRT-7 (5). The RRT-7 can be run as a stand-alone instrument or in a fully automated system with in-line HPLC or UV analysis. The physical dimension of a stand-alone system is about 30 " $\times 15^{\prime \prime} \times 42$ " (width $\times$ depth $\times$ height), shown in Figure 2. The RRT-7 uses a "dry heat" system for temperature control and does not need a sink. It can run up to 24 samples at one time with the use of two 12-sample carousels. Plastic stoppers that sit on the individual test tubes cover the sample containers to minimize medium evaporation.

To release two lots of product using a conventional Apparatus 7, heating, temperature control, and cleaning of two water baths for two instruments are required, while it takes only one Logan RRT-7 to release two lots of product, and the "dry heat" system on Logan RRT-7 is not only maintenance free, but also consumes less electricity. Thus, the unique design of the Logan Model RRT-7 allows higher sample capacity, lowers operation cost, and requires less bench space per analysis compared with the conventional USP Apparatus 7. In this study, we used both Varian Apparatus 7 and Logan RRT-7 to perform the in vitro drug release test of scopolamine transdermal systems.

\section{Materials and Methods}

\section{Reagents and Apparatus}

Scopolamine base reference standard was qualified inhouse. Scopolamine transdermal systems were made by Aveva Drug Delivery Systems, Inc., Miramar, FL, USA. Acetonitrile and ammonium acetate were purchased from Fisher Scientific, Pittsburgh, PA, USA. Distilled water was made inhouse. The two USP Apparatus 7 instruments were made by Varian, Inc., Palo Alto, CA, USA and Logan Instruments Corp., Somerset, NJ,USA. The HPLC-UV was made by Agilent Technologies, Inc., Santa Clara, CA, USA. The chromatographic data acquisition software was Waters Millennium 4.0 from Waters, Milford, MA, USA.

\section{Preparation of Solutions and Standards}

Since water was the dissolution medium selected for the Reference Listed Drug Product (Transderm Scop ${ }^{\circledR}$ ), the dissolution medium used for this test was distilled water that was degassed by heating to $41^{\circ} \mathrm{C}$, filtered through a 0.45- $\mu \mathrm{m}$ HVLP membrane filter under vacuum, and stirred for an additional 5 minutes after the filtration was completed. The mobile phase was a mixture of $10 \mathrm{mM}$ ammonium acetate and acetonitrile in the ratio of 50/50 $(\mathrm{v} / \mathrm{v})$ that was filtered through a $0.45-\mu \mathrm{m} \mathrm{HVLP}$ membrane filter and degassed through an on-line degasser before use. The stock standard solution contained about $0.20 \mathrm{mg} / \mathrm{mL}$ of scopolamine base dissolved in the dissolution medium. The working standards contained about $0.06 \mathrm{mg} / \mathrm{mL}$ of scopolamine base diluted from the stock standard solution with the dissolution medium.

\section{Dissolution Conditions}

The medium volume per vessel was $20 \mathrm{~mL}$. The temperature was $32.0 \pm 0.3^{\circ} \mathrm{C}$. The stroke depth of the reciprocating disks was $2-3 \mathrm{~cm}$. The dipping (or stroking) speed was 45 dips per minute. The number of transdermal systems per test was 12. All other dissolution conditions are listed in Table 1.

\section{Sample Preparation}

The drug release testing station was heated, and the bath temperature was verified to be within $32.0 \pm 0.3^{\circ} \mathrm{C}$. Twenty milliliters of the dissolution medium was transferred into each of 12 test tubes with a $20-\mathrm{mL}$ volumetric pipet and allowed to equilibrate to $32.0 \pm 0.3^{\circ} \mathrm{C}$. The test tubes were covered to minimize evaporation once the medium was transferred and during the course of the drug release test. A double-sided tape was applied to the surface of each reciprocating disk. A single scopolamine transdermal system was attached to each disk with the backing side against the double-sided tape after the protective layer of the doublesided tape was removed. The transdermal system was care- 
fully placed to avoid air bubble formation. The liner of the transdermal system was removed prior to the testing. At each time of pull, the solutions in the test tubes were reserved for HPLC analysis, and another 12 test tubes containing $20.0 \mathrm{~mL}$ of the fresh dissolution medium in each were used. The motion of dipping was continued in the test tubes with the fresh dissolution medium. This process was repeated until the sample solutions were collected at the last time point, 72 hours.

\section{Chromatographic Analysis}

The HPLC column was Waters $\mu$ Bondapak C18,3.9 × 150 $\mathrm{mm}, 10-\mu \mathrm{m}$ particle size. The mobile phase flow rate was 1.0 $\mathrm{mL} / \mathrm{min}$. The column temperature was $25^{\circ} \mathrm{C}$. The injection volume was $15 \mu \mathrm{L}$. The detection wavelength was $215 \mathrm{~nm}$. The typical retention time of scopolamine was about 2.5-3.5 $\mathrm{min}$. The run time per injection was $5 \mathrm{~min}$. The analytical method was validated for its accuracy, precision, linearity, limit of quantitation, specificity, solution stability, and robustness that included varying the mobile phase buffer concentration, aqueous-to-organic mobile phase ratio, and column temperature. The detailed method validation results are not presented here.

\section{Calculation}

The scopolamine concentration, $C_{n}(\mathrm{mg} / \mathrm{mL})$, in a sample solution at each time point was calculated by using eq 1 , and the cumulated release of scopolamine at each time point in percent of label claim, $\% \mathrm{~L} C_{t}$, was calculated by using eq 2:

$$
\begin{aligned}
C_{n} & =\frac{R_{\text {sample }}}{R_{\text {BSTD }}} \times C_{\text {WSTD }} \\
\% C_{t}= & \frac{\sum_{x=1}^{n}\left(C_{x} \times V\right)}{L C} \times 100
\end{aligned}
$$

where $n$ is $1,2,3,4,5,6$, or $7 ; \mathrm{R}_{\text {sample }}$ is the peak area of scopolamine in a sample; $\mathrm{R}_{\mathrm{BSTD}}$ is the average peak area of all scopolamine bracketing standards including those for system suitability; $C_{\text {WSTD }}$ is scopolamine concentration in the working standard solution; $C_{x}$ is scopolamine concentration

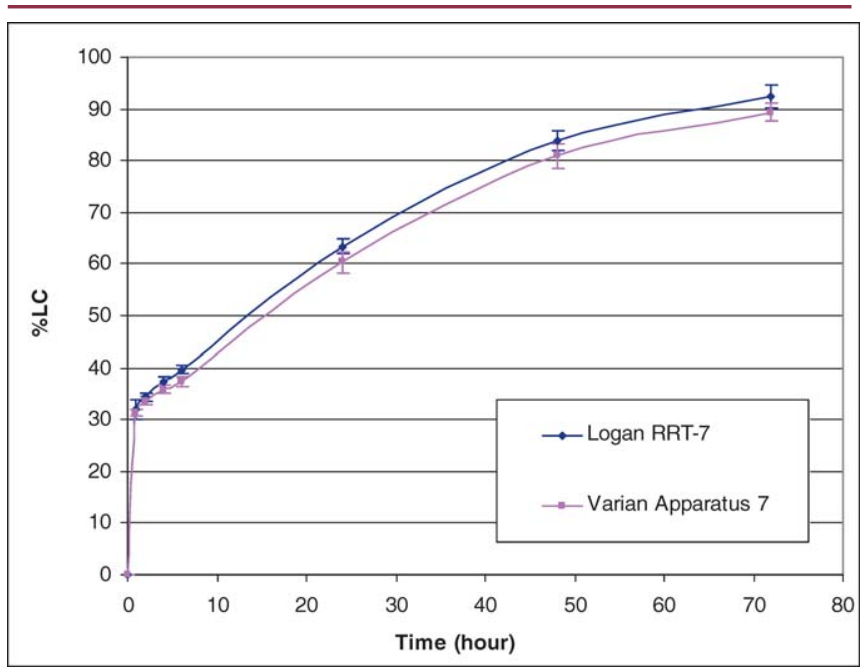

Figure 3. Comparison of drug release profiles of product Lot $X$ obtained from different manufacturers of USP Apparatus 7. Each data point is the average of 12 values, and the error bar indicates the standard deviation of each data point.

at $x^{\text {th }}$ time point in $\mathrm{mg} / \mathrm{mL} ; x$ is $1,2, \ldots, \mathrm{n}$; V is the volume of medium in each test tube; and $L C$ is the label claim of the product in $\mathrm{mg}$.

\section{Results and Discussion}

The comparison of dissolution profiles of solid oral dosage forms can be achieved by using the similarity factor $f_{2}$ through the mathematical treatment of dissolution data

(6). Two dissolution profiles are considered similar when $\mathrm{f}_{2} \geq$ 50. We extended the application of the similarity factor $f_{2}$ to the comparison of release profiles for transdermal dosage forms, although the similarity factor $\mathrm{f}_{2}$ was referred for the solid oral dosage forms. The values of the similarity factor $f_{2}$ for various experiments are listed in Table 2. For each drug release test, twelve units of scopolamine transdermal systems were consumed.

\section{Comparison of Drug Release by Using Apparatus 7 from Two Manufacturers}

The drug release test on scopolamine transdermal systems, Lot X, was performed using USP Apparatus 7 made by both VanKel/Varian and Logan Instruments Corp. The

Table 2. $f_{2}$ Values from Comparison Experiments.

\begin{tabular}{|l|l|l|c|}
\hline \multicolumn{2}{|c|}{ Experiment } & $\mathbf{f}_{\mathbf{2}}$ Value \\
\hline Drug release using the Apparatus 7 from Varian & vs & Drug release using the Apparatus 7 from Logan & 80 \\
\hline Drug release by using Carousel A from Logon RRT-7 & vs & Drug release by using Carousel B from Logon RRT-7 & 96 \\
\hline Test 1 using Logan RRT-7 & vs & Test 2 using Logan RRT-7 & 74 \\
\hline
\end{tabular}




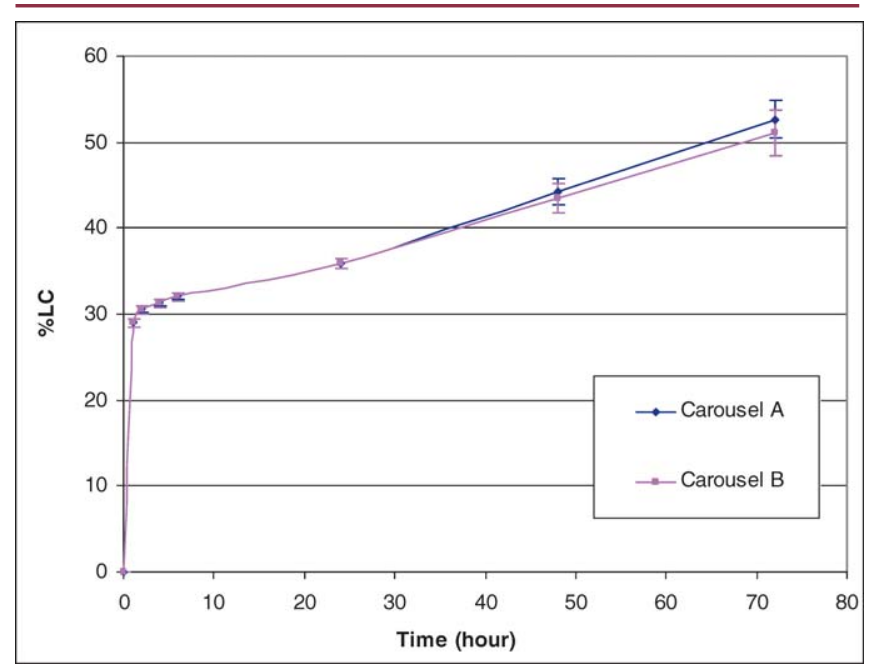

Figure 4. Comparison of drug release profiles of product Lot Y obtained from two carousels of Logan RRT-7. Each data point is the average of 12 values, and the error bar indicates the standard deviation of each data point.

relative standard deviation of 12 units for any time point was within $1.9-3.3 \%$ and $1.9-5.8 \%$ for Varian Apparatus 7 and Logan RRT-7, respectively. Although the time gap between the two tests on the same lot of scopolamine transdermal systems was about 2 months, the drug release profiles were similar, as shown in Figure 3. Furthermore, the $\mathrm{f}_{2}$ factor value of 80 also confirms that the two drug release profiles are similar.

\section{Comparison of Drug Release by Using the Two Carousels from Logon RRT-7}

The drug release test of scopolamine transdermal system, Lot $Y$, was simultaneously performed by using both carousels ( $A$ and B) from the Apparatus 7 made by Logan when the product was freshly made. The relative standard deviation for any time point was within $1.2-4.1 \%$ and 1.5-5.2\% for Carousels A and B, respectively. The drug release profiles from the two carousels were almost completely superimposed as shown in Figure 4, which indicate close similarity between the two carousels, also confirmed by an $\mathrm{f}_{2}$ factor value of 96 . Note that the amount of drug release at each time pull was lower than that at the same time pull from the experiment using apparatus from different manufacturers; this was because Lot $Y$ was processed differently from Lot $\mathrm{X}$ used in the previous experiment.

\section{Repeatability of Logan RRT-7}

The drug release test on scopolamine transdermal systems, Lot $Y$, was performed later on different days by using the same USP Apparatus 7 made by Logan Instruments Corp. The drug

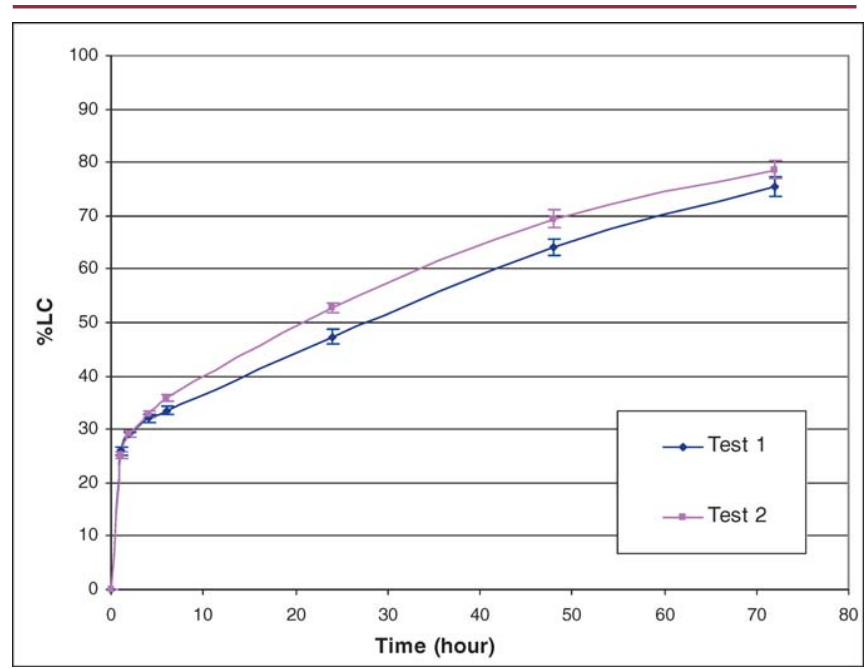

Figure 5. Comparison of drug release profiles of product Lot $Y$ obtained on different days by Logan RRT-7. Each data point is the average of 12 values, and the error bar indicates the standard deviation of each data point.

release profiles are presented in Figure 5. The relative standard deviation for any time point was within $1.8-2.6 \%$ and $1.8-2.7 \%$ for Test 1 and Test 2, respectively. Due to the product change with time, there might be differences in the products that contributed to the slight difference in profiles. Nevertheless, the $\mathrm{f}_{2}$ factor value of 74 demonstrated that the two drug release profiles were similar.

\section{Conclusion}

There are differences in design between the USP Apparatus 7 made by VanKel/Varian and the Model RRT-7 made by Logan Instruments Corp. The Logan design allows higher sample capacity, lowers operation cost, and requires less bench space per analysis compared with the conventional USP Apparatus 7. The drug release profiles of scopolamine transdermal systems obtained by using both conventional Apparatus 7 and Logan RRT-7 were similar. Our study demonstrated good day-to-day repeatability of dissolution profiles using the Logan RRT-7 system, as well as good intrasystem repeatability using the two 12-sample carousels.

\section{Acknowledgment}

The authors appreciate the service engineers from Logon Instruments Corp. for their technical support.

\section{References}

1. Zarmi, S.; Roa, R.; Löbenberg, R. Current perspectives in dissolution testing of conventional and novel dosage forms. Int. J. Pharm. 2007, 328, 12-21.

2. Transderm Scop ${ }^{\circledR}$. http://www.transdermscop.com/ site2/prescribe.html (accessed March 2007). 
3. United State Pharmacopeia and National Formulary USP 29-NF 24; The United States Pharmacopeial Convention, Inc:: Rockville, MD, 2006.

4. Varian, Inc. Apparatus 3 / Apparatus 7 Operator's Manual. http://www.varianinc.com/ image/vimage/docs/products/dissolution/shared/om70-9017J.pdf (accessed March 2007).
5. Industry News: Logan Instruments Corp. launches RRT 7 and Workstation 24/7 at AAPS, San Antonio, TX. Dissolution Technol. 2006, 13 (4), 32.

6. Dissolution Testing of Immediate Release Solid Oral Dosage Forms; Guidance for Industry; U.S. Department of Health and Human Services, Food and Drug Administration, U.S. Government Printing Office:Washington, DC, August 1997. 